Research Article

\title{
Identification of Macrophage Polarization-Related Genes as Biomarkers of Chronic Obstructive Pulmonary Disease Based on Bioinformatics Analyses
}

\author{
Yalin Zhao $\mathbb{D}$, Meihua Li, Yanxia Yang, Tao Wu, Qingyuan Huang, Qinghua Wu, \\ and Chaofeng Ren
}

Respiratory and Critical Care Medicine, Kunming First People's Hospital, Kunming, Yunnan Province, China

Correspondence should be addressed to Yalin Zhao; 39031381@qq.com and Chaofeng Ren; 593988414@qq.com

Received 31 March 2021; Accepted 4 June 2021; Published 21 June 2021

Academic Editor: Nagarajan Raju

Copyright (C) 2021 Yalin Zhao et al. This is an open access article distributed under the Creative Commons Attribution License, which permits unrestricted use, distribution, and reproduction in any medium, provided the original work is properly cited.

\begin{abstract}
Objectives. Chronic obstructive pulmonary disease (COPD) is characterized by lung inflammation and remodeling. Macrophage polarization is associated with inflammation and tissue remodeling, as well as immunity. Therefore, this study attempts to investigate the diagnostic value and regulatory mechanism of macrophage polarization-related genes for COPD by bioinformatics analysis and to provide a new theoretical basis for experimental research. Methods. The raw gene expression profile dataset (GSE124180) was collected from the Gene Expression Omnibus (GEO) database. Next, a weighted gene coexpression network analysis (WGCNA) was conducted to screen macrophage polarization-related genes. The differentially expressed genes (DEGs) between the COPD and normal samples were generated using DESeq2 v3.11 and overlapped with the macrophage polarization-related genes. Moreover, functional annotations of overlapped genes were conducted by Database for Annotation, Visualization and Integrated Discovery (DAVID) Bioinformatics Resource. The immune-related genes were selected, and their correlation with the differential immune cells was analyzed by Pearson. Finally, receiver operating characteristic (ROC) curves were used to verify the diagnostic value of genes. Results. A total of 4922 coexpressed genes related to macrophage polarization were overlapped with the 203 DEGs between the COPD and normal samples, obtaining 25 genes related to COPD and macrophage polarization. GEM, S100B, and GZMA of them participated in the immune response, which were considered the candidate biomarkers. GEM and $S 100 B$ were significantly correlated with marker genes of B cells which had a significant difference between the COPD and normal samples. Moreover, GEM was highly associated with the genes in the $\mathrm{PI} 3 \mathrm{~K} / \mathrm{Akt} / \mathrm{GSK} 3 \beta$ signaling pathway, regulation of actin cytoskeleton, and calcium signaling pathway based on a Pearson correlation analysis of the candidate genes and the genes in the B cell receptor signaling pathway. PPI network analysis also indicated that GEM might participate in the regulation of the PI3K/Akt/GSK3 $\beta$ signaling pathway. The ROC curve showed that GEM possessed an excellent accuracy in distinguishing COPD from normal samples. Conclusions. The data provide a transcriptome-based evidence that GEM is related to COPD and macrophage polarization likely contributes to COPD diagnosis. At the same time, it is hoped that in-depth functional mining can provide new ideas for exploring the COPD pathogenesis.
\end{abstract}

\section{Introduction}

Chronic obstructive pulmonary disease (COPD), an inflammatory disease of the lung mainly caused by smoking tobacco cigarettes and environmental exposure from burning biomass fuels or air pollution $[1,2]$, has become a major health problem around the world [3]. It has been reported that COPD killed about 3 million people in 2016, and the mortal- ity rates of COPD are still growing [4]. Worse, although COPD has long been considered treatable, the diagnosis and treatment of COPD in the clinical practice remain to be improved $[5,6]$. Currently, the diagnosis of COPD mainly depends on the use of spirometry by identifying pulmonary dysfunction [7], which is full of limitations, such as the detection of the early stage of COPD. Moreover, although some blood-related biomarkers were related to exacerbations, 
progression, or mortality of COPD, it was unknown whether they could be selected as the diagnostic biomarkers [8]. On the other hand, even if the traditional treatments including lung volume reduction surgery for COPD have made great strides, there were still some uncontrollable complications [9]. In addition, even if some pulmonary rehabilitation programs, such as exercise training, education, and behavior change followed by patient-tailored therapies have been regarded as promising measures for improving the COPD patients, a few patients still cannot gain the benefit from the exercise training [10]. Hence, it is very wishful for further parsing the molecular mechanism and recognizing the novel biomarkers of COPD.

With the development of bioinformatics, recent studies have revealed that COPD susceptibility is associated with the genes' expression [11-14]. For example, it has been suggested that the expression of B3GNT, LAF4, and ARHGEF10 can predict the frequent exacerbations of COPD [11]. Moreover, IL6 and SOCS3 also have been suggested to play a key role in COPD and can be used as the therapeutic targets of COPD [12]. Moreover, Zhang et al. also found that TLR2 and CD79A may be used as the potential biomarkers for the clinical severity of COPD and related to the inflammatory responses of COPD [13]. More importantly, it has been revealed that COPD can be subdivided into three molecular subtypes based on the gene expression profiles of 213 COPD-related genes [14]. Therefore, bioinformatics analysis of gene expression profiling may contribute to screening novel biomarkers of COPD resulting in improving the treatment of COPD.

Increasing evidences have proposed that macrophage which is an important effector cell for the innate immune response plays a crucial role in COPD [15-17]. A recent study has demonstrated that COPD patients exhibit more macrophages in the bronchial alveolar lavage fluid, along the airways and lung parenchyma compared to the normal samples $[15,16]$. Besides, Traves et al. also found more blood monocyte-derived macrophages into the airspaces of COPD [17]. More importantly, emerging evidences showed that macrophage polarization may be associated with COPD [18-20]. For example, a previous study has suggested an increase in proinflammatory M1 macrophages in the small airways of COPD compared to controls, but a reciprocal decrease in M2 macrophages [18]. On the other hand, the results of Eapen et al. suggested that lncRNA MIR155HG modulated the progression of COPD by inducing the granulocyte-macrophage colony-stimulating factor-mediated M1/M2 macrophage polarization [19]. In addition, Shaykhiev et al. suggested that the reprogramming for alveolar macrophage polarization likely contributes to COPD pathogenesis [20]. Nevertheless, the research focusing on the molecular regulation mechanism of macrophage polarization in COPD is inadequate.

With the development and extensive applications of bioinformatics, the weighted gene coexpression network analysis (WGCNA) has become an important and effective method to screen hub genes for complex disease [21]. In recent years, WGCNA has been performed to identify genes which are related to clinical features in many diseases, such as stroke [22] and schizophrenia [23]. Moreover, all of these researches suggested that screening biomarkers for diagnosis and treatment of complex disease by WGCNA was effective.

In the present study, we firstly downloaded the raw gene expression profile dataset which included COPD samples and normal samples from the Gene Expression Omnibus (GEO) database (https://www.ncbi.nlm.nih.gov/geo/query/ acc.cgi). Next, WGCNA was carried out to screen macrophage polarization-related genes. Finally, we identified hub genes as the novel diagnostic biomarkers for COPD and further analyzed their molecular mechanisms in COPD, which will contribute to the treatment of COPD.

\section{Materials and Methods}

2.1. Dataset Acquisition. The raw gene expression profile dataset (GSE124180, only peripheral blood samples) was collected from the GEO database [24]. The dataset based on GPL16791 platform included 6 COPD samples and 15 normal samples.

2.2. WGCNA. The weighted gene coexpression network analysis was performed using WGCNA R package (v1.69) in the COPD and normal samples [25]. A total of 35 macrophage polarization genes (Supplementary Table 1) from the MsigDB database (https://www.gsea-msigdb.org/ gsea/msigdb) were considered different traits to investigate the coexpressed genes related to macrophage polarization genes. Soft thresholding was then applied by raising correlation values to a power of 14 to amplify disparity between strong and weak correlations. The soft thresholding power was chosen to achieve approximately scale-free network topology, as recommended for biological networks $[26,27]$. The resulting signed adjacency matrix was used to calculate topological overlap matrix (TO), which was then hierarchically clustered with (1-TO) as a distance measure.

Genes were then assigned into coexpression modules by dynamic tree cutting algorithm requiring minimal module size of 100 genes [28]. The modules with highly correlated eigengenes (correlation above 0.6) were merged. Module eigengene (ME) is the first principal component of the gene expression values within a module and is used to summarize the module's expression. The Pearson correlation between each gene and $\mathrm{ME}$ was then calculated. This value is called module membership (MM) and represents how close a particular gene is to a module. Finally, each gene was assigned to a module for which it had the highest MM. The module with the highest absolute value of the correlation coefficient with the traits was chosen as the key module for subsequent analysis.

\subsection{Differential Expression Analysis and the Candidate Gene} Identification. The comparison of differential expressions between the COPD and normal samples was generated using DESeq2 v3.11 [29]. The $p$ value $<0.05$ and $\left|\log _{2} \mathrm{FC}\right|>0.8$ were considered the cutoff value. The candidate genes were identified by overlapping the DEGs and the genes in the key module, getting 25 genes related to COPD and macrophage polarization, and their expressions were displayed by a heatmap in the COPD and normal samples. 

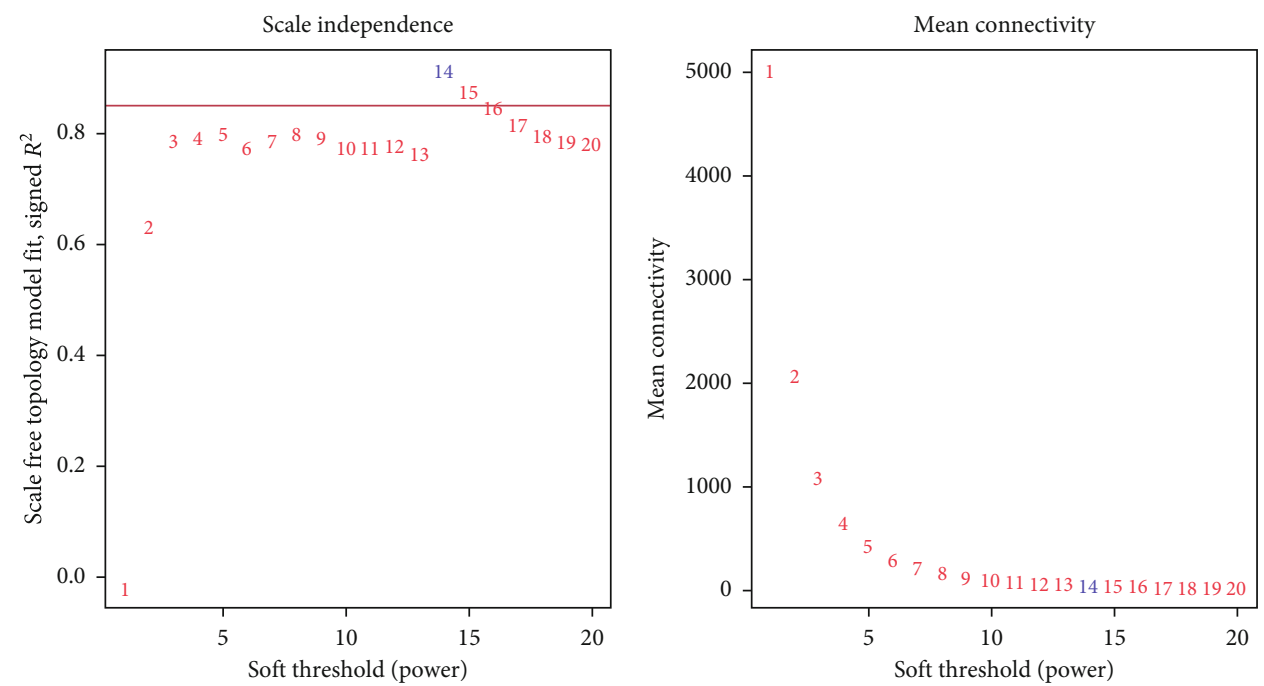

(a)

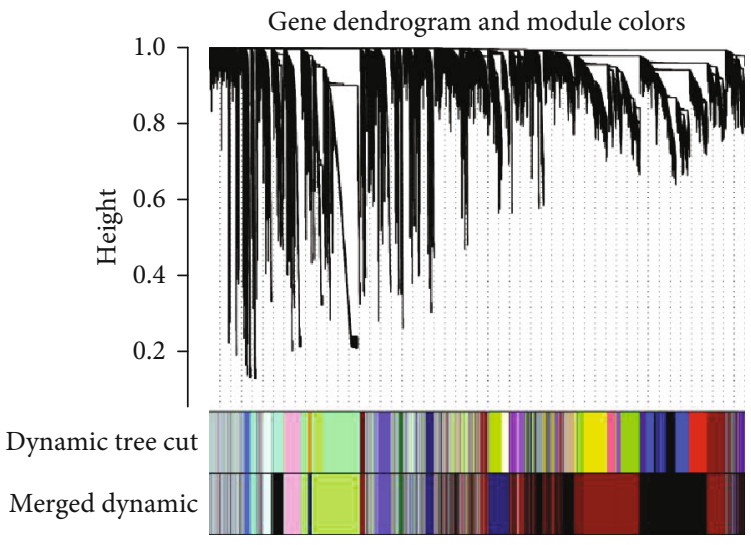

(b)

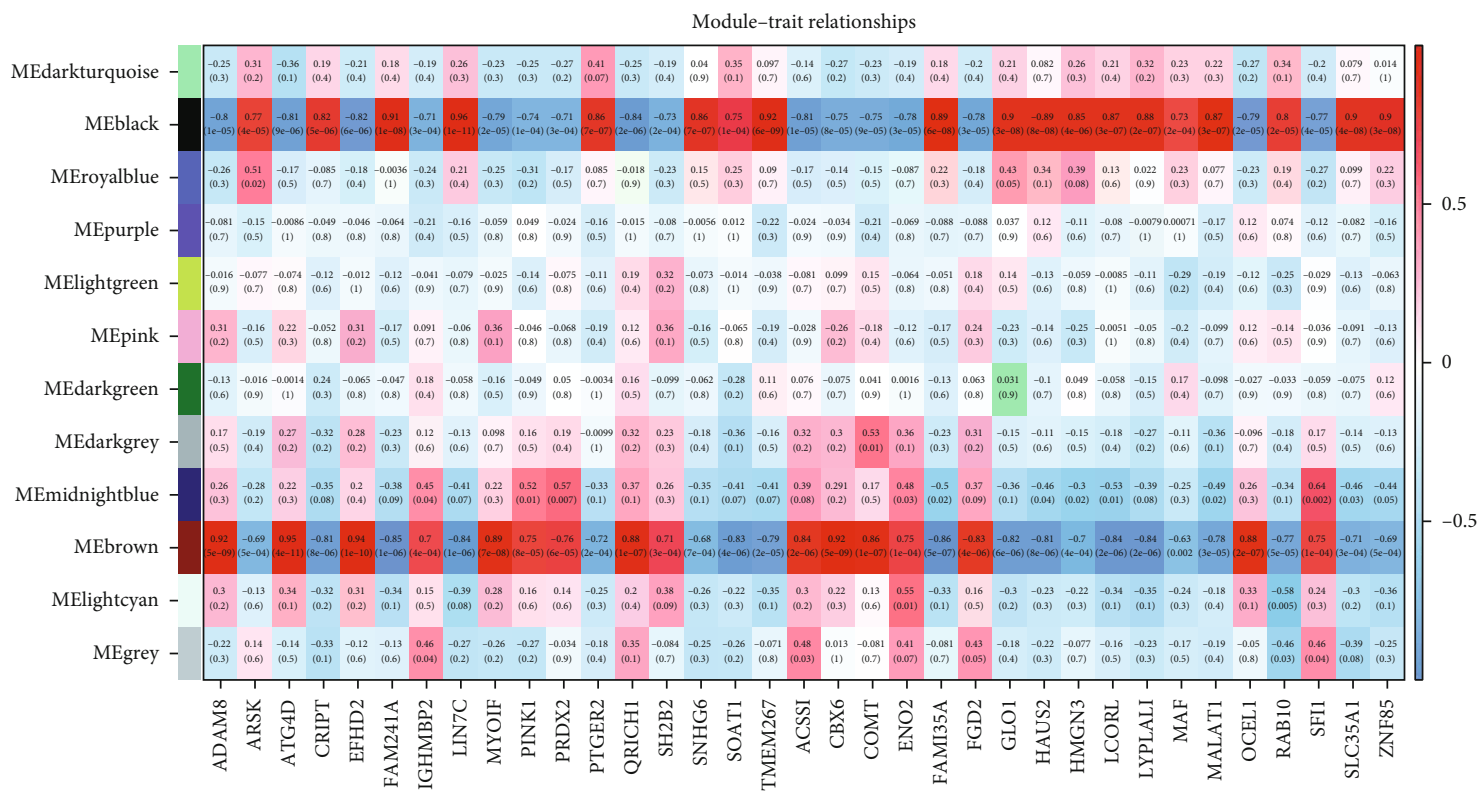

(c)

FIGURE 1: Construction weighted gene coexpression network and identification of modules related to the markers of macrophage polarization. (a) Determination the optimal soft threshold to conform to the scale-free distribution. (b) Dendrogram of genes clustered based on the highly correlated eigengenes (correlation above 0.6 ). 

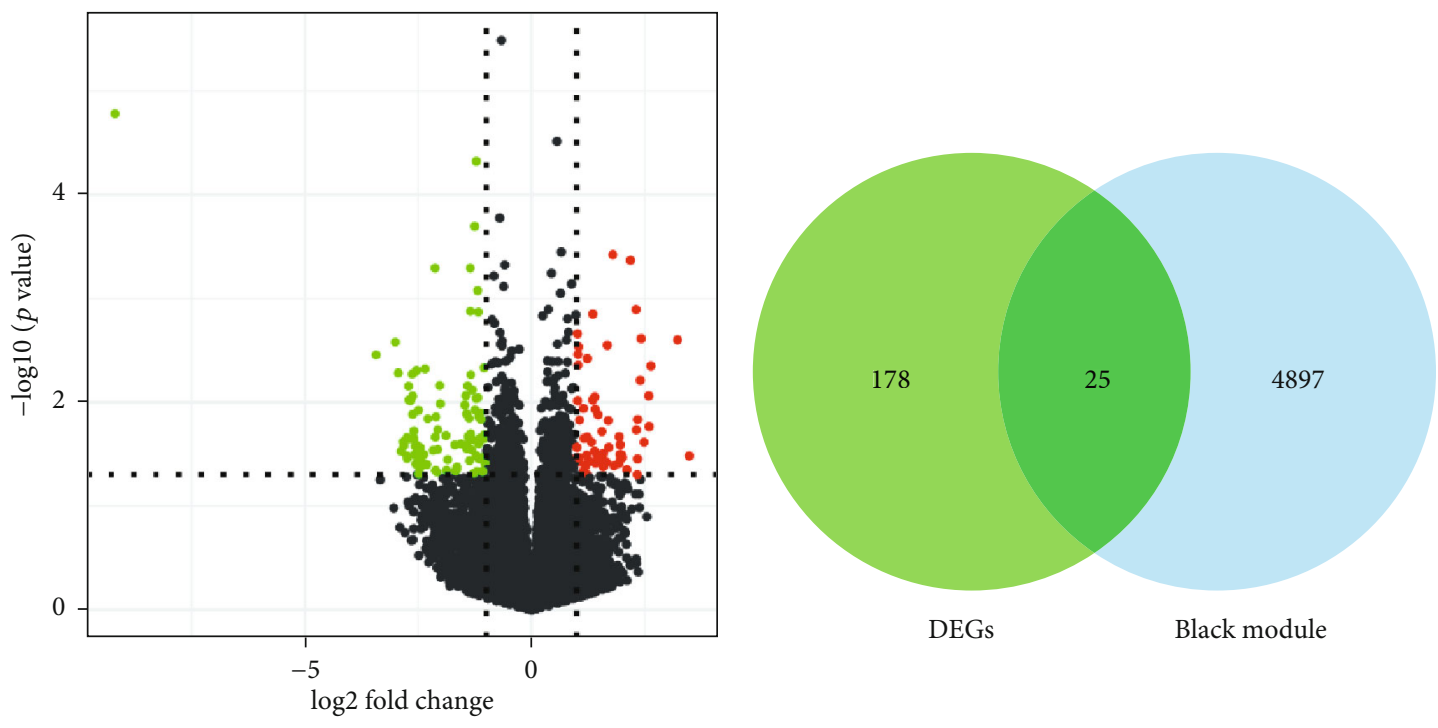

Significant

- Down

- No

- Up

(a)

(b)

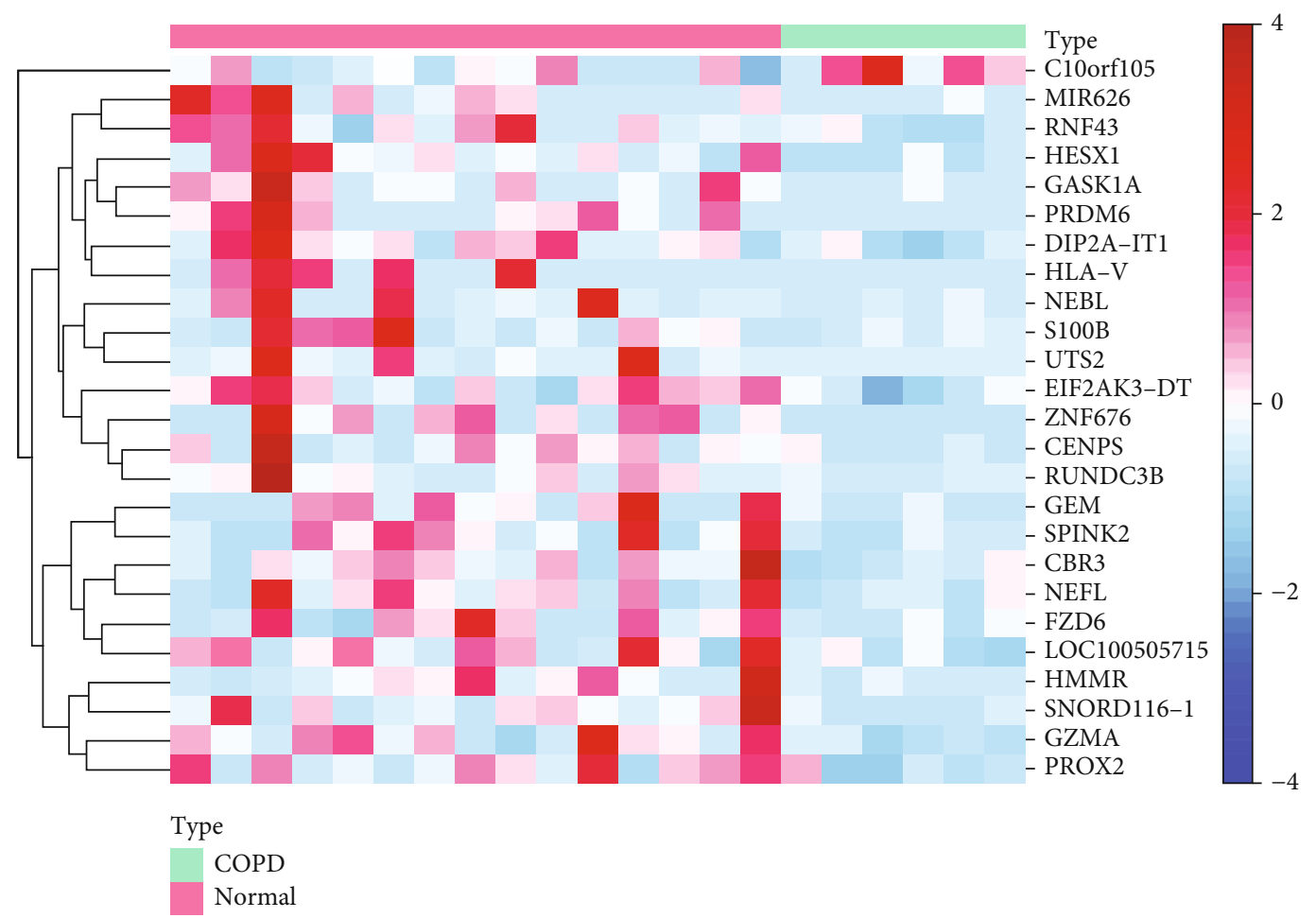

(c)

FIGURE 2: Identification of genes associated with COPD and macrophage polarization. (a) Volcano plot showed the DEGs between COPD samples and normal samples. (b) Overlapping genes between DEGs and macrophage polarization-related genes. (c) Heatmap exhibited the differential expression of the candidate genes between COPD samples and normal samples.

2.4. The Functional Annotations and Correlation Analysis of the Candidate Genes. DAVID bioinformatics resource (v6.8), an online website (https://david.ncifcrf.gov/), was devoted to conduct the functional annotations of the 25 genes [30]. The immune-related genes (as the candidate genes) were selected from the 25 genes, and Pearson analysis was used to investigate the correlation of the candidate genes and the differential immune cells between the COPD and normal samples, as well as marker genes and pathways of differential immune cells. 
TABLE 1: The results of GO functional annotation and KEGG pathways for the 25 candidate genes.

\begin{tabular}{lc}
\hline ID & Biological process \\
\hline CBR3 & $\begin{array}{c}\text { GO:0042376 } \sim \text { phylloquinone catabolic process, GO:0050890 } \\
\text { cognition, GO:0055114 oxidation-reduction process }\end{array}$
\end{tabular}

GO:0001736 establishment of planar polarity, GO:0001843 neural tube closure, GO:0001942 hair follicle development, GO:0007186 G-protein coupled receptor signaling pathway, GO:0007223 Wnt signaling pathway, calcium modulating pathway, GO:0007275 multicellular organism development, GO:0030168 platelet activation, GO:0033278 cell

FZD6

NEFL

ZNF676 proliferation in midbrain, GO:0035567 non-canonical Wnt signaling pathway, GO:0035880 embryonic nail plate morphogenesis, GO:0042472 inner ear morphogenesis,

GO:0043433 negative regulation of sequence-specific DNA binding transcription factor activity, GO:0060071 Wnt signaling pathway, planar cell polarity pathway, GO:0090090 negative regulation of canonical Wnt signaling pathway, GO:1904693 midbrain morphogenesis

GO:0000165 MAPK cascade, GO:0000226 microtubule cytoskeleton organization, GO:0008089 anterograde axonal transport, GO:0008090 retrograde axonal transport, GO:0009636 response to toxic substance, GO:0014012 peripheral nervous system axon regeneration, GO:0019896 axonal transport of mitochondrion, GO:0021510 spinal cord development, GO:0021766 hippocampus development, GO:0021987 cerebral cortex development, GO:0031133 regulation of axon diameter, GO:0033693 neurofilament bundle assembly, GO:0040011 locomotion, GO:0043434 response to peptide hormone, GO:0043524 negative regulation of neuron apoptotic process, GO:0043547 positive regulation of GTPase activity, GO:0045105 intermediate filament polymerization or depolymerization, GO:0045109 intermediate filament organization, GO:0048812 neuron projection morphogenesis, GO:0050772 positive regulation of axonogenesis, GO:0050885 neuromuscular process controlling balance, GO:0051258 protein polymerization, GO:0051412 response to corticosterone, GO:0061564 axon development, GO:1903935 response to sodium arsenite, GO:1903937 response to acrylamide

GO:0006351 transcription, DNA-templated, GO:0006355 regulation of transcription, DNA-templated

GO:0000122 negative regulation of transcription from RNA polymerase II promoter, GO:0006351 transcription, DNA-

PROX2 templated, GO:0030182 neuron differentiation, GO:0045944 $\sim$ positive regulation of transcription from RNA polymerase II promoter, GO:0055007 cardiac muscle cell differentiation

HMMR GO:0000086 G2/M transition of mitotic cell cycle, GO:0030214 hyaluronan catabolic process

GO:0006955 immune response, GO:0007067 mitotic nuclear division, GO:0007165 signal transduction, GO:0007166 cell surface receptor signaling pathway, GEM GO:0007264 small GTPase mediated signal transduction, GO:0051276 chromosome organization, GO:0051310 metaphase plate congression

GO:0006351 transcription, DNA-templated, GO:0007420 brain development,GO:0030916 otic vesicle formation, HESX1 GO:0043584 nose development, GO:0045892 negative regulation of transcription, DNA-templated, GO:0048853 forebrain morphogenesis, hsa00590: arachidonic acid metabolism, hsa00980: metabolism of xenobiotics by cytochrome P450, hsa01100: metabolic pathways

hsa04310: Wnt signaling pathway, hsa04390: Hippo signaling pathway, hsa04550: signaling pathways regulating pluripotency of stem cells, hsa04916: melanogenesis, hsa05166:HTLV-I infection, hsa05200: pathways in cancer, hsa05205: proteoglycans in cancer, hsa05217: basal cell carcinoma

hsa05014: amyotrophic lateral sclerosis (ALS),

hsa04512: ECM-receptor interaction

hsa04550: signaling pathways regulating pluripotency of stem cells 
TABLE 1: Continued.

\begin{tabular}{lc}
\hline ID & Biological process \\
\hline & GO:0006351 transcription, DNA-templated, GO:0022008 \\
neurogenesis, GO:0034968 histone lysine methylation, \\
PRDM6 \\
GO:0045892 negative regulation of transcription, DNA- \\
templated, GO:0051151 $\sim$ negative regulation of smooth \\
muscle cell differentiation
\end{tabular}

RUNDC3B

GO:0007409 axonogenesis, GO:0007417 central nervous system development, GO:0007611 learning or memory, GO:0007613 memory, GO:0008283 cell proliferation, GO:0008284 positive regulation of cell proliferation, GO:0008360 regulation of cell shape, GO:0043065 positive regulation of apoptotic process, GO:0043123 positive regulation of I-kappaB kinase/NF-kappaB signaling,

S100B

CENPS

C10orf105

(0.006915 apoptotic process, GO:0006955 immune regulation of endodeoxyribonuclease activity, GO:0043065

GZMA positive regulation of apoptotic process, GO:0043392 negative regulation of DNA binding, GO:0051354 negative regulation of oxidoreductase activity, GO:0051603 proteolysis involved in cellular protein catabolic process

NEBL GO:0071691 cardiac muscle thin filament assembly GO:0016055 Wnt signaling pathway, GO:0016567 protein ubiquitination, GO:0030178 negative regulation of Wnt RNF43 signaling pathway, GO:0038018 $\sim$ Wnt receptor catabolic process, GO:0042787 protein ubiquitination involved in ubiquitin-dependent protein catabolic process, GO:0072089 stem cell proliferation,

GO:0002176 male germ cell proliferation, GO:0007286 spermatid development, GO:0009566 fertilization,

SPINK2 hsa03460: Fanconi anemia pathway

hsa04080: neuroactive ligand-receptor interaction 
TABLE 1: Continued.

\begin{tabular}{|c|c|c|}
\hline ID & Biological process & KEGG pathway \\
\hline UTS2 & 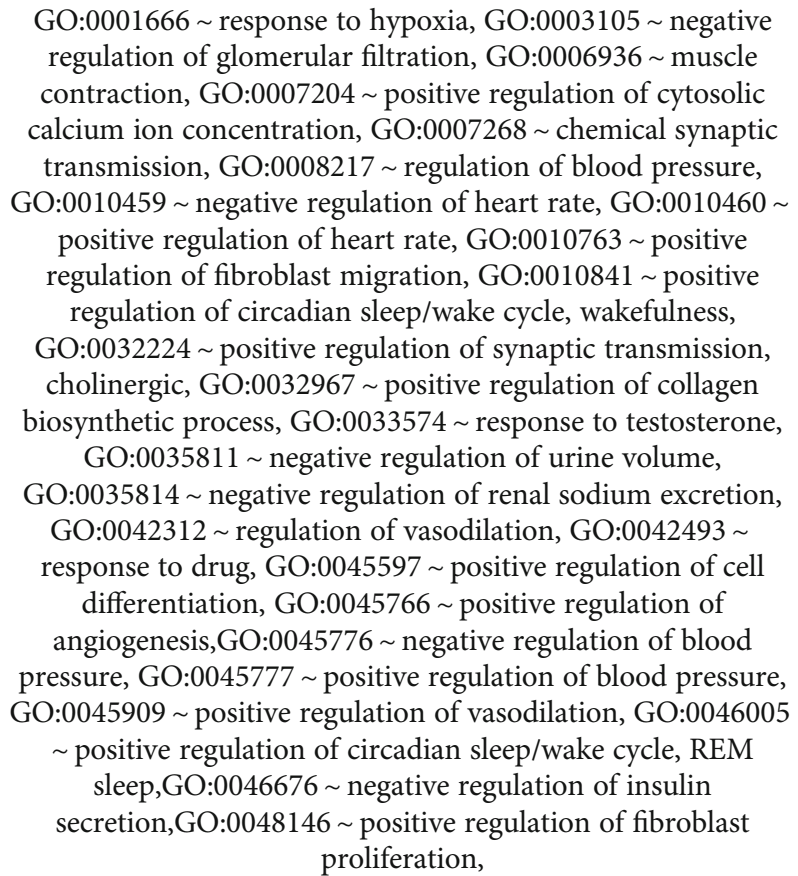 & \\
\hline
\end{tabular}

2.5. Construction of PPI Network and LASSO Model. To further evaluate the functions of the candidate genes, the PPI network was constructed by IPA (v01-18-05) to explore the gene-related pathways. Besides, a LASSO model was established by the candidate genes using the glmnet $\mathrm{R}$ package (v4.0-2) [31]. The ROC curve was drawn by the pROC R package (v1.16.12) and used to analyze the ability of the model and gene to distinguish the COPD and normal samples [32]. The higher the area under the curve (AUC), the stronger the predictive capacity.

\section{Results}

3.1. Selection of Coexpressed Genes Related to Macrophage Polarization. The coexpression network of the 21 samples was divided into 26 modules. We had chosen the soft threshold power 14 (marked with blue) to define the adjacency matrix based on the criterion of approximate scale-free topology (Figure 1(a)). The modules whose eigengenes were correlated above 0.6 would be merged (Figure 1(b)). Subsequently, the analysis of the relationship of the modules and the traits suggested that multiple modules (black, brown, and midnight blue) were closely related to one or more traits, especially the black module, which was the one with the highest correlation coefficient strongly associated with the macrophage polarization (Figure 1(c)). The black module contained 4922 eignegenes (Supplementary Table 2). Thus, the black module was selected as the coexpressed genes related to macrophage polarization.

3.2. Candidate Gene Identification. A total of 203 DEGs was generated between the COPD and normal samples and shown by a volcano plot (Figure 2(a)), including 82 upregulated genes (Supplementary Table 3) and 121 downregulated genes (Supplementary Table 4). The DEGs were overlapped with the 4922 eignegenes in the black module (Figure 2(b)), obtaining 25 genes as the candidate genes related to COPD and macrophage polarization. The candidate genes were listed in Supplementary Table 5, and their expression was shown by a heatmap which exhibited an obvious difference between the COPD and normal samples (Figure 2(c)). Almost all of the candidate genes in COPD samples had lower expressions compared to that in normal samples, except for C10orf105 which was highly expressed in COPD samples, suggesting that it enabled these candidate genes related to macrophage polarization to commendably distinguish COPD from normal samples.

3.3. Functional Annotations of the Candidate Genes. To further explore the functions of the candidate genes, functional annotations were conducted by DAVID. Only 18 of the 25 candidate genes received corresponding functional annotations. As shown in Table 1, GEM, S100B, and GZMA participated in the immune response, which were considered the candidate genes for subsequent analyses. Macrophages are traditional innate immunocytes, as well as being involved into adaptive immune response. Macrophage polarization is a dynamic process, macrophages switch reversibly between M1 (proinflammatory) phenotype and M2 (anti-inflammatory) phenotype which is implicated in the immune response [33]. Therefore, we next would attempt to investigate the correlation between the candidate genes related to macrophage polarization and immunity. 

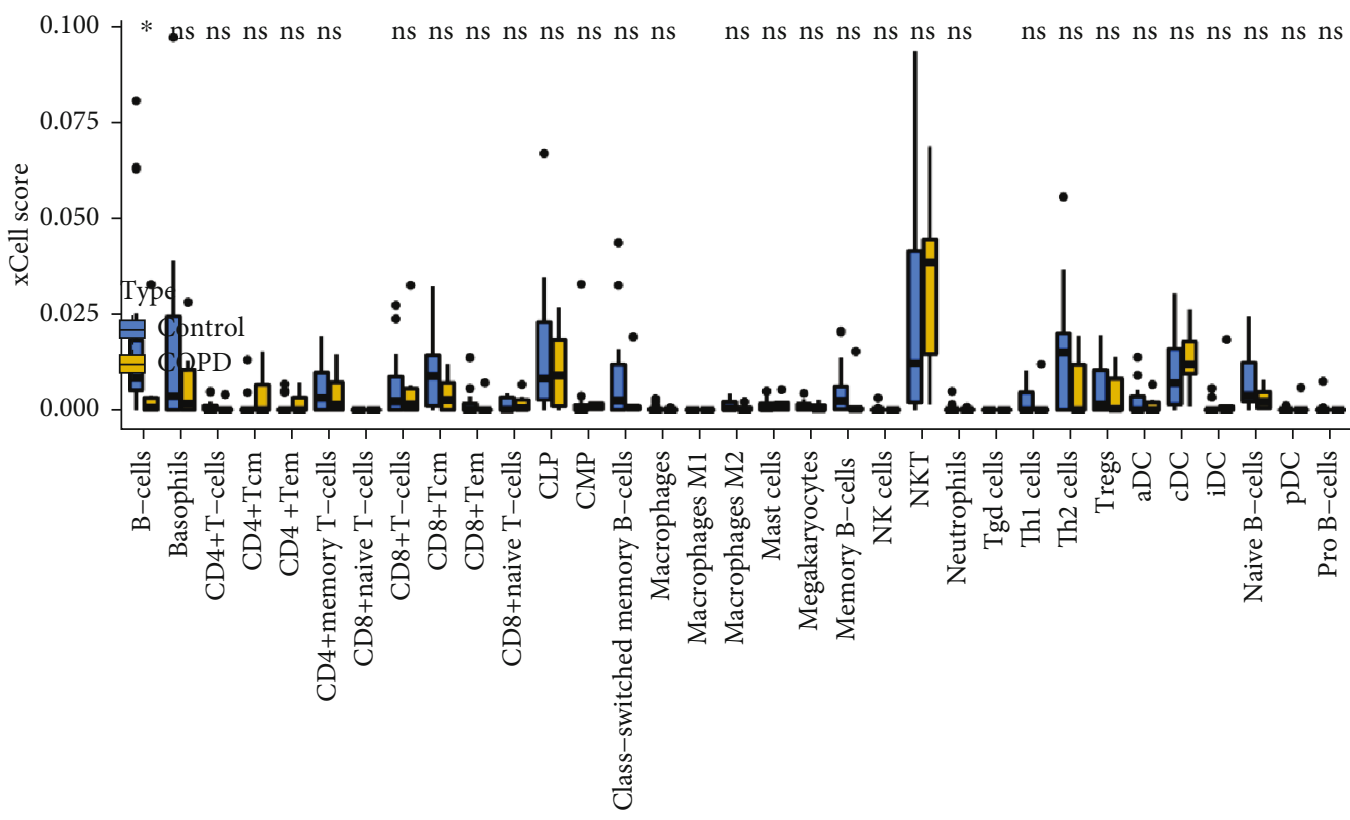

Immunocyte

Type

Control

官 $\mathrm{COPD}$
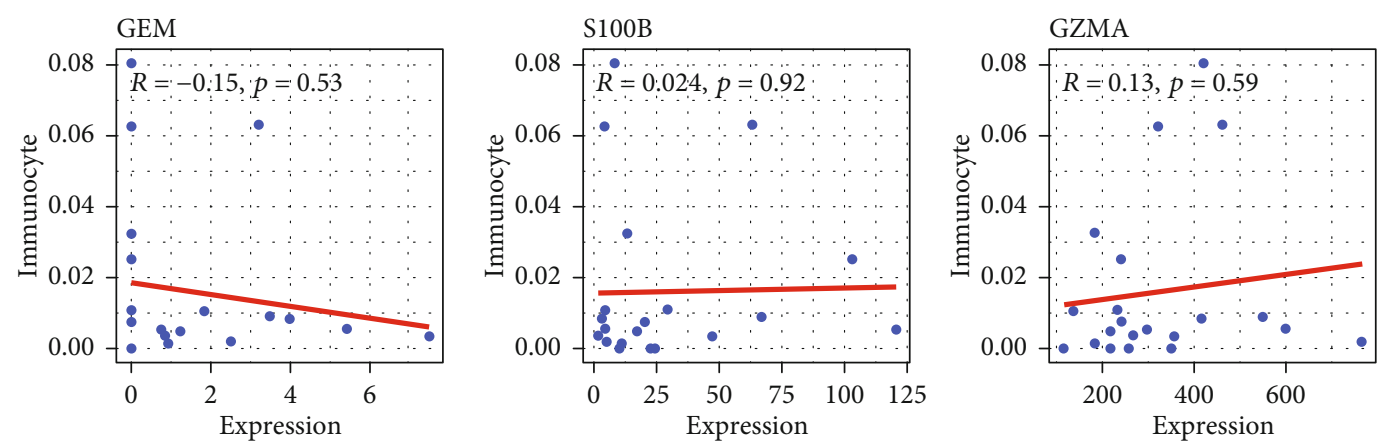

(b)

Figure 3: Continued. 


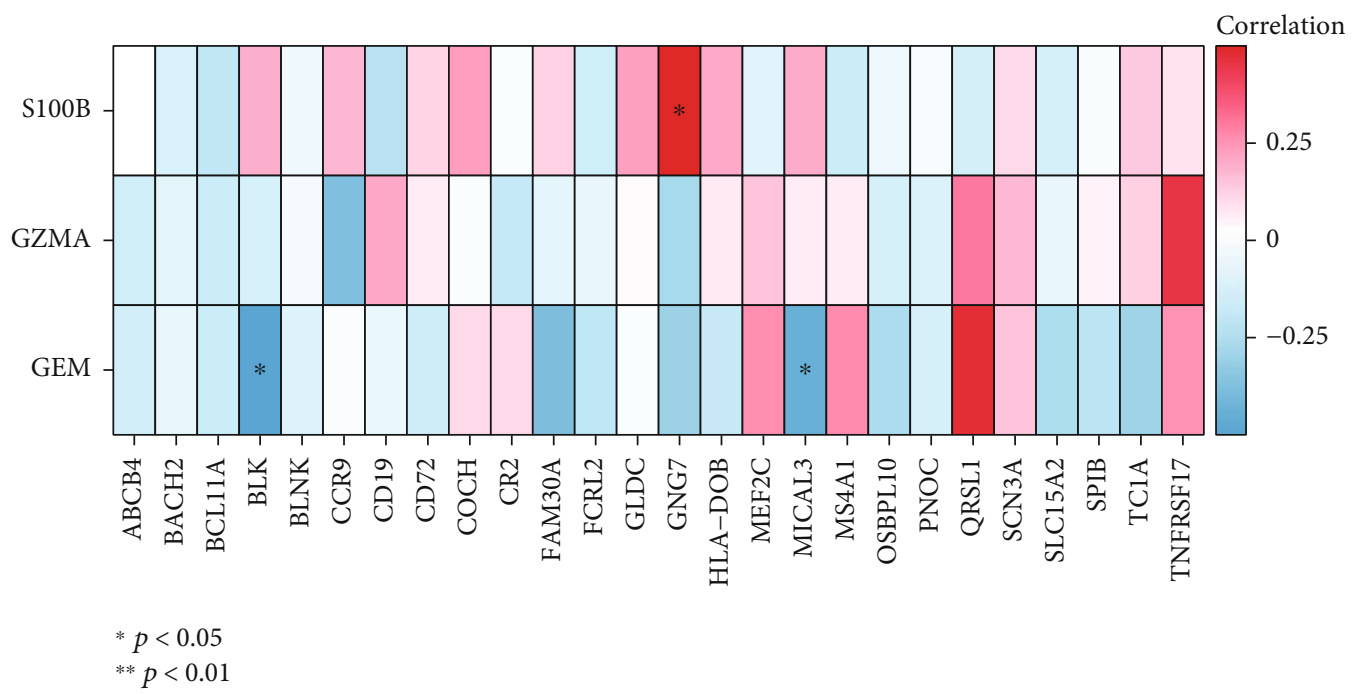

(c)

FIgURE 3: Correlation between candidate biomarkers and immune-infiltrated cells. (a) Box plot displayed the immune cell infiltration levels between COPD samples and normal samples. (b) Bubble plots showed the correlation between candidate biomarkers and immune-infiltrated cells. (c) Heatmap exhibited the correlation between candidate biomarkers and the marker genes of B cells.

It was worth noting that some genes were involved in the process of neuronal activity, such as CBR3, FZD6, NEFL, PROX2, PRDM6, S100B, and GZMA. NEFL was found to be involved in amyotrophic lateral sclerosis- (ALS-) related signaling pathways at the same time. Here, we speculated that when COPD occurred, these genes were regulated by macrophage polarization, which in turn affected motor neuron activity, resulting in motor neuron atrophy, and motor weakness and atrophy (like ALS). This may be the reason why patients with COPD often accompany unexplained skeletal muscle atrophy [34].

3.4. Relationship of the Candidate Genes with Immune Cells. To explore the correlation between the candidate genes and immunity, we analyzed the score of immune cells in the COPD and normal samples, showing that only the score of $B$ cells had a significant difference between the COPD and normal samples. Compared with normal samples, score of the B cells was significantly reduced in the COPD samples ( $p$ value $<0.05$, Figure 3(a)). But, GEM, S100B and GZMA were not significantly associated with the score of $B$ cells (all $p$ value $>0.05$, Figure $3(\mathrm{~b})$ ), which might be caused by too small samples. To further study the correlation between the candidate genes and $\mathrm{B}$ cells, we downloaded the 26 marker genes from a literature with Immunology Journal [35]. Correlation of the candidate genes with the 26 marker genes were displayed by a heatmap (Figure 3(c)), suggesting that GEM was significantly negatively correlated with two marker molecules (BLK and MICAL3, all $p$ values < 0.05 ), $S 100 B$ was significantly positively correlated with one marker molecule (GNG7, $p$ value < 0.05 ), while GZMA was not correlated with any marker molecules. Thus, it can be seen that GEM and S100B may participate in the progression of COPD via regulating activity of $\mathrm{B}$ cells.
3.5. Involvement of the Candidate Genes in the B Cell Receptor Signaling Pathway. The B cell receptor signaling pathway is of great importance for B cell survival and proliferation. The B cell receptor aberrantly expressed on B cells contributes to the multiple disease pathogenesis, and its signaling pathway is currently the target of several therapeutic strategies [36]. Therefore, we investigated the correlation of the candidate genes with the genes in the B cell receptor signaling pathway which were obtained from the Kyoto Encyclopedia of Genes and Genomes (KEGG) database (Figure 4(a)). The result indicated that GEM was highly associated with the genes in the PI3K/Akt/GSK3 $\beta$ signaling pathway ( $A K T$ and $G S K 3 B$, all $p$ values $<0.05)$, regulation of the actin cytoskeleton (BCR, LYN, and RAC, all $p$ values $<0.05$ ), and the calcium signaling pathway (SYK, PLCG2, NFAT5, and NFATC1, all $p$ values $<0.05$ ) and might be partially related to the NF- $\kappa \mathrm{B}$ signaling pathway (only MALT1, IKBKB, $N F K B 2$, and $I K B$, all $p$ values $<0.05)$. Besides, no correlation between $G E M$ and the MAPK signaling pathway was found (Figure 4(b)). Genes and pathways significantly related to GEM were marked in Figure 4(a). These results indicated that GEM might regulate $\mathrm{B}$ cell activity through the PI3K/Akt/GSK3 $\beta$ signaling pathway, regulation of actin cytoskeleton, and calcium signaling pathway, as well as the $\mathrm{NF}-\kappa \mathrm{B}$ signaling pathway.

3.6. Construction of PPI Network. To further verify GEM-regulated genes in pathways, all genes were used to construct a PPI network based on the screening of DEGs (Figure 5(a)), in which GEM, S100B, and GZMA were signed. In the network, red represents upregulated genes, and green represents downregulated genes. Gray indicates that the element is a predicted element. The solid line represents the proteins that have been experimentally confirmed to have physical contact with each other, and the dotted line represents that the 


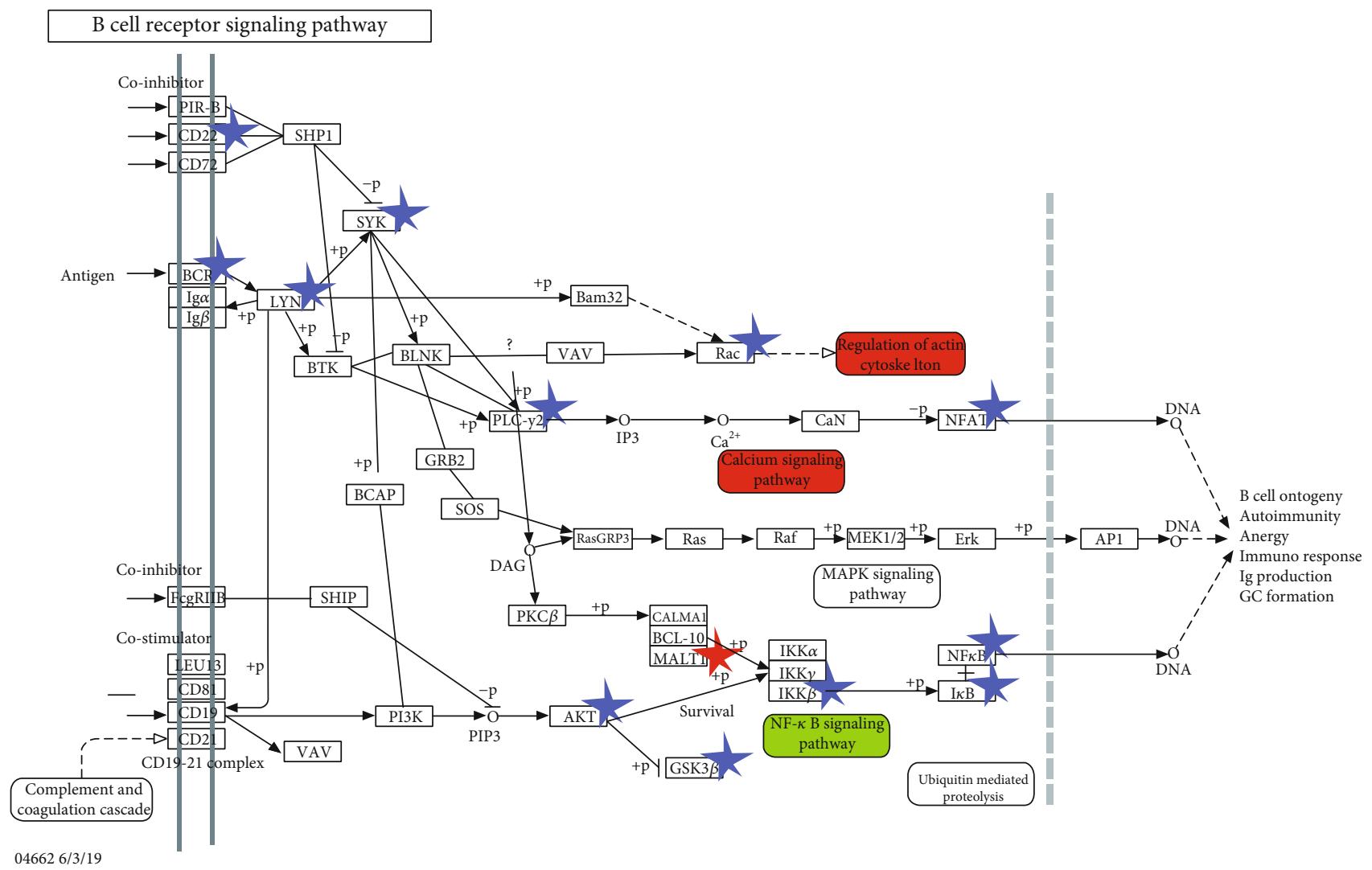

(c) Kanehisa laboratories

(a)

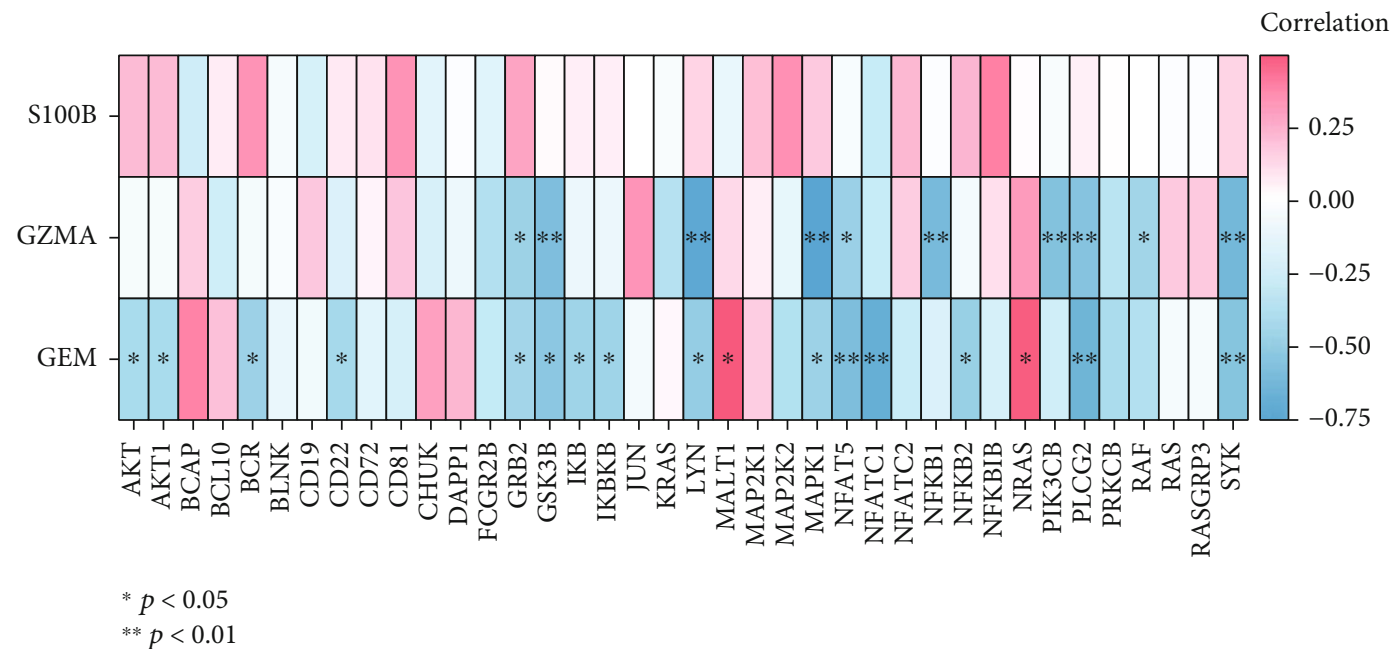

(b)

FIgURE 4: Association between candidate biomarkers and B cell receptor signaling pathway. (a) Regulation network of the B cell receptor signaling pathway. (b) Heatmap exhibited the correlation between candidate biomarkers and the genes in the B cell receptor signaling pathway.

interaction between the proteins is physically combined without being confirmed by the interaction experiment. The color of the line has no special meaning, and the arrow represents the object of action. Then, to show the interactions of GEM with other genes more clearly, we individually selected the PPI network of GEM. As shown in Figure 5(b), GEM was associated with Akt which was a gene that inter- acted with the most genes. This was consistent with our above results that GEM might participate in the regulation of the PI3K/Akt/GSK3 $\beta$ signaling pathway.

3.7. Diagnostic Value of GEM. To investigate the accuracy of the genes for distinguishing the COPD samples from normal samples, we firstly constructed a LASSO model using the 


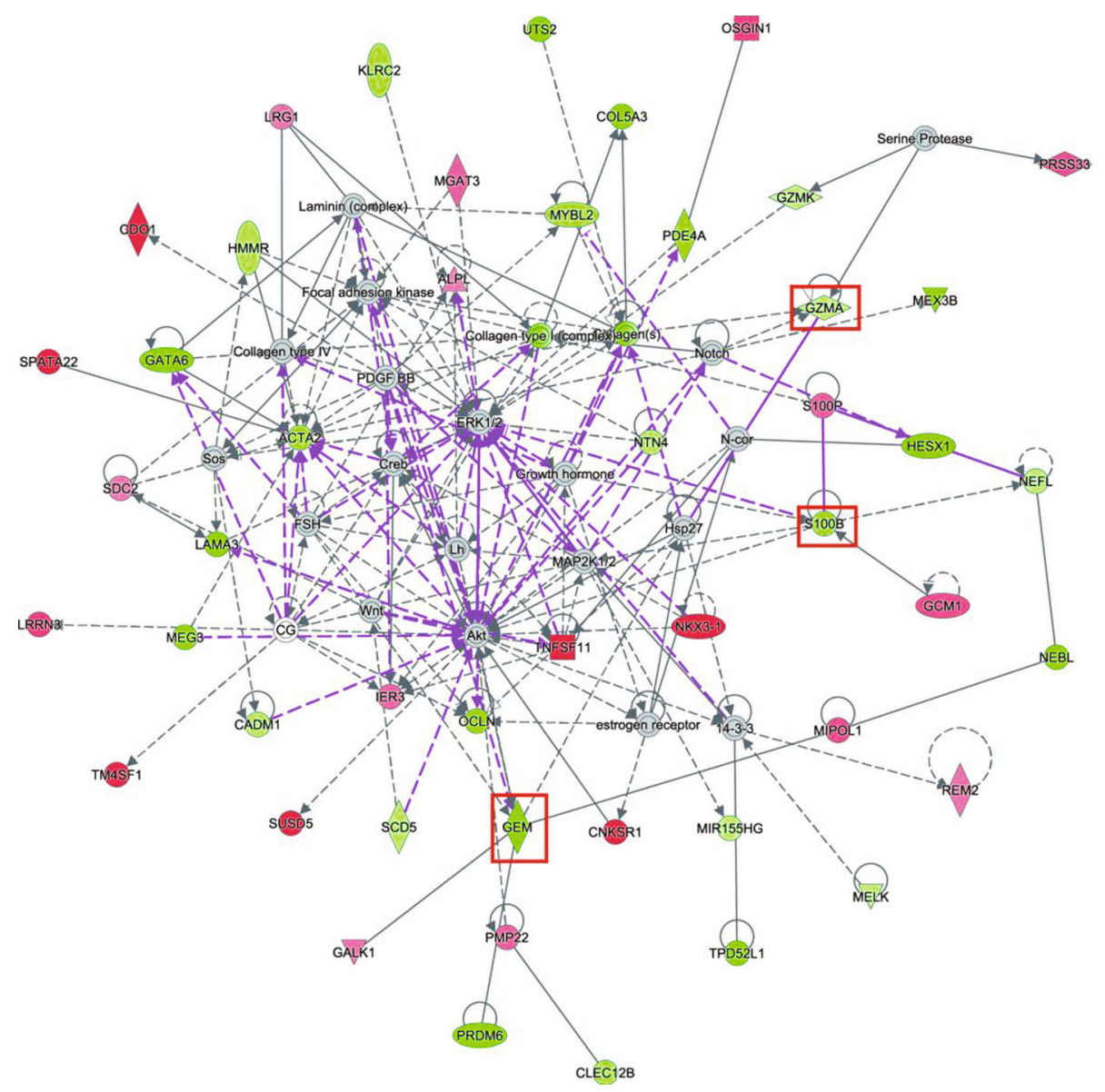

(a)

FIgUre 5: Continued. 


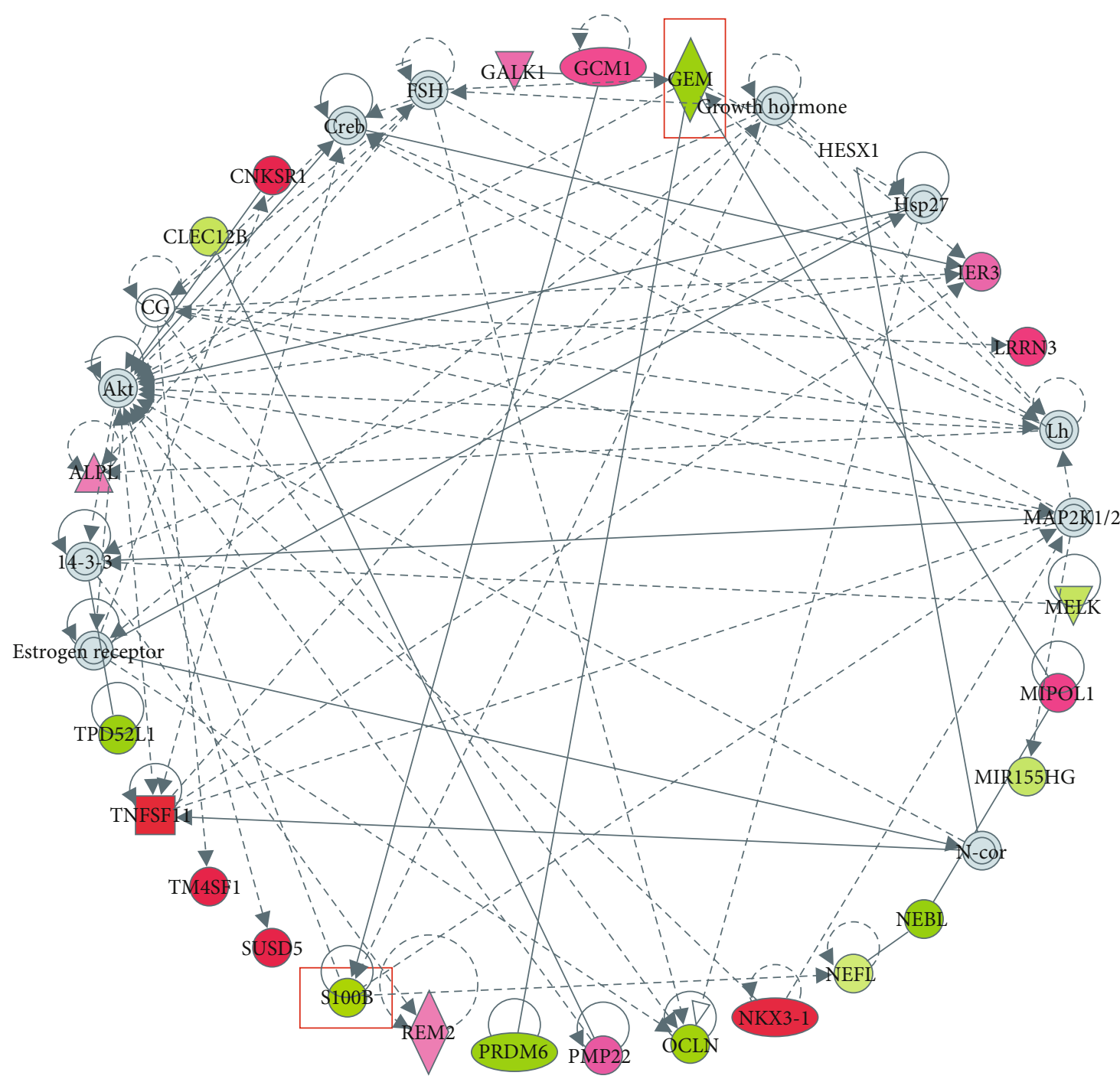

(b)

FIGURE 5: Construction of PPI network. (a) PPI network for candidate biomarkers and other DEGs. (b) PPI network for GEM and other DEGs.

three candidate genes (Figure 6(a)). The accuracy of the LASSO model was verified by the ROC curve, and the AUC was 0.717 (Figure 6(b)). Meanwhile, we plotted the other ROC curves with only GEM, and the AUC was $0.844>$ 0.717 (Figure 6(c)). These results suggested that GEM, rather than the three candidate genes, possessed an excellent accuracy in distinguishing COPD from normal samples.

\section{Discussion}

COPD, characterized by persistent airflow obstruction, is an irreversible and preventable disease [37]. Although from 1990 to 2015, the death rate of COPD has gone down [38, 39], COPD will become the third leading cause of death worldwide in 2030 [40]. However, the diagnosis of COPD mainly relied on the spirometry values and clinical symptoms, which is full of continual underdiagnosis [41]. Hence, it is urgent to further study the molecular mechanism and screen novel and reliable biomarkers to improve the diagnosis of COPD.

Notably, there are some researches that focused on the GSE124180 dataset. For example, the authors uploaded the GSE124180 dataset mainly compared the correlation of gene expression across COPD-relevant tissues, including largeairway epithelium, alveolar macrophage, and peripheral blood, and explored the biological functions of overlapped genes among three tissues [42]. On the other hand, Baschal et al. compared the gene expressions across the lower airway, sinus, and middle ear tissues using the GSE124180 dataset and other data [43]. Different from these two researches, our study mainly focused on the role of macrophage polarization in COPD and identification of macrophage polarization-related genes as the biomarkers of COPD based on the peripheral blood of the GSE124180 dataset.

In our study, we identified 25 genes related to COPD and macrophage polarization, which were considered candidate genes for further analysis (Figure 2(b), Supplementary 

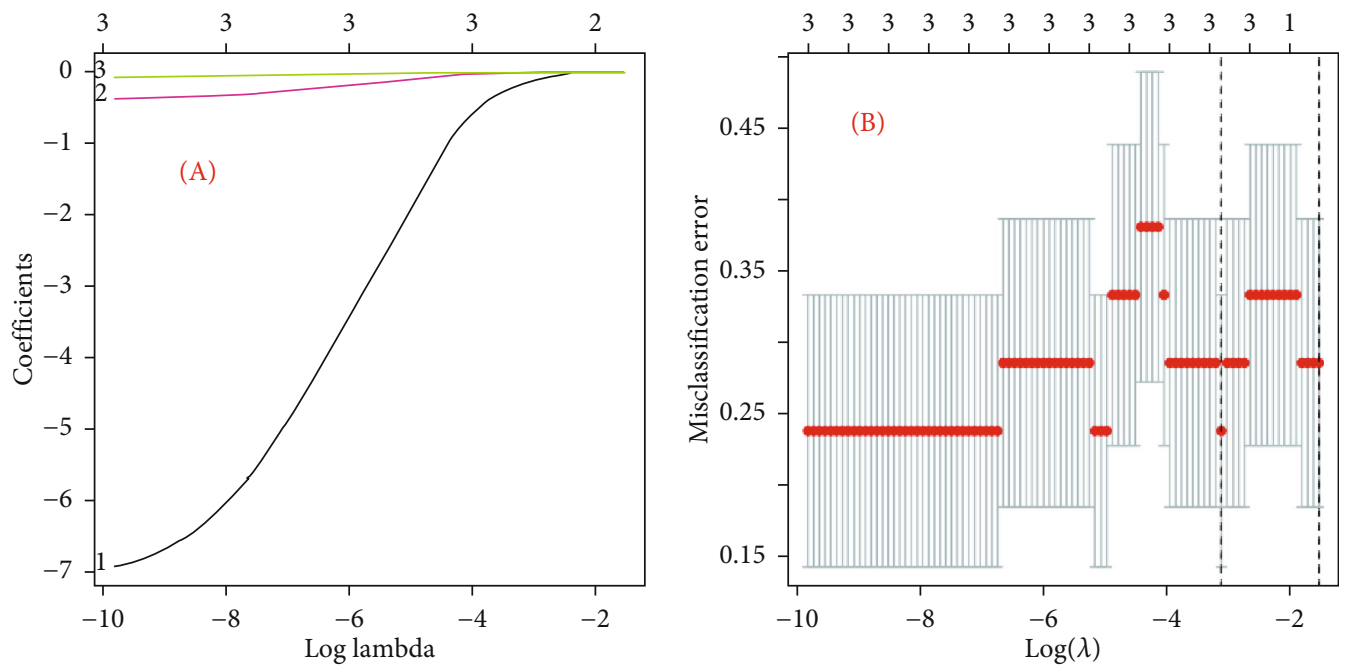

(a)

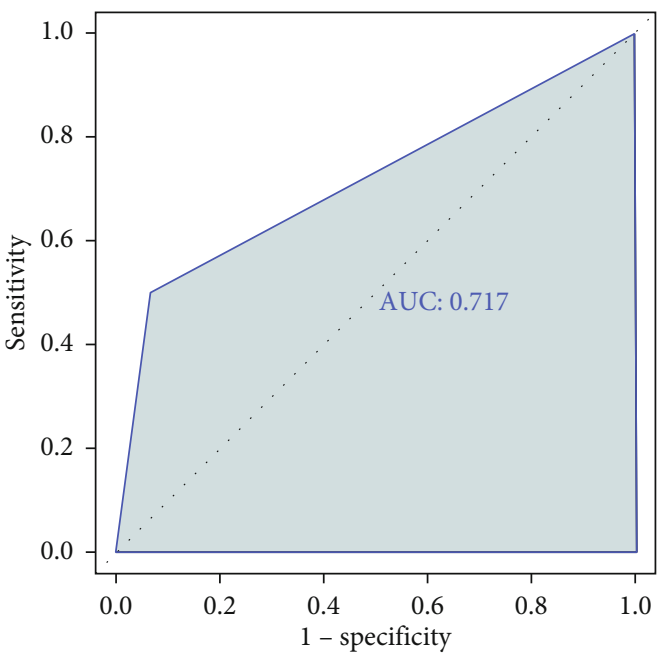

(b)

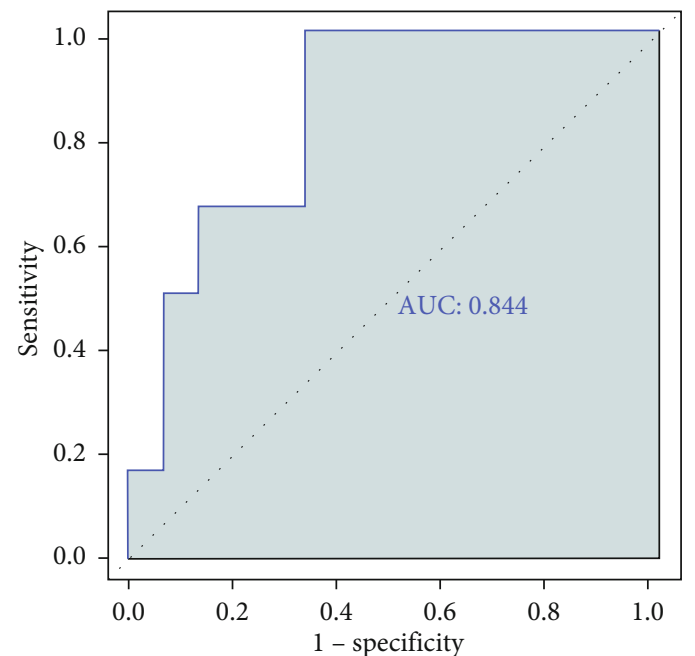

(c)

FIGURE 6: Investigation of the accuracy of the candidate biomarkers for distinguishing the COPD samples from normal samples. (a) Construction of the LASSO model based on 3 candidate biomarkers: (A) image showed the log (lambda) value of the 3 candidate biomarkers and (B) image showed the distribution of the log (lambda) value in the LASSO model. (c) ROC curve for the LASSO model. (d) ROC curve for the GEM.

Table 5). Moreover, to further explore the functions of the 25 candidate genes, we performed the GO annotation and KEGG enrichment analyses. We found that for the biological process, these 25 candidate genes mainly involved in immune response, signal transduction, cell proliferation, negative regulation of skeletal muscle cell differentiation, and so on (Table 1). The analysis of KEGG pathway enrichment showed that these 25 genes mainly related to metabolic pathways, Wnt signaling pathway, Hippo signaling pathway, amyotrophic lateral sclerosis, ECM-receptor interaction, and so on (Table 1). Increasing evidence has revealed that obesity can affect the morbidity of COPD [44, 45]. Thus, we speculated that metabolic pathways may play an important role in COPD. In addition, it has been suggested that Wnt signaling is associated with the inflammatory response in lung alveolar epithelial cells [46]. Especially, in macrophages, Wnt5a can induce inflammatory response via FZD5 and Wnt3a can mediate anti-inflammatory effects by the $\mathrm{Wnt} / \beta$-catenin signaling pathway [47]. Therefore, theses 25 genes might be associated with the COPD.

Clearly, the results of functional annotations for the 25 genes suggested that GEM, S100B, and GZMA participated in the immune response and were selected as the hub candidate genes (Table 1). GEM, a small GTP binding, is a member of RAS superfamily of monomeric G-proteins [48]. It has been suggested that GEM is involved in signal transduction [49]. Although it was not reported that GEM was related to COPD in recent studies, it has been suggested that GEM is related to the skeletal muscle for type 2 diabetes [49]. Thus, we speculated that GEM might be related to skeletal muscle dysfunction of COPD. $S 100 B$, a member of the $S 100$ protein family for $\mathrm{Ca}^{+}$-binding proteins of the EF-hand type, is increasingly being studied in the central nervous system 
[50]. Moreover, a recent study has proved that $S 100 B$ is associated with the reparative process in acute muscle injury and muscular dystrophy by regulating the M1/M2 macrophage levels [51]. In addition, it has been also found that $S 100 B$ upregulated TNF- $\alpha$ and M1 markers in RAW264.7 macrophages [52]. $S 100 B$ could participate in the FGFR1mediated inflammatory response in osteoarthritis [53]. Besides, $S 100 B$ was necessary for the progression of vascular immune responses in neonatally infected rat brains [54]. GZMA, a member of the serine protease family, may play a key role for the pathophysiology of different inflammatory disorders by acting as a proinflammatory mediator [55-57]. Garzón-Tituaña et al. also found that GZMA could serve as a proinflammatory mediator in macrophages by inducing the TLR4-dependent expression of IL-6 and TNF- $\alpha$ [58]. On the other hand, it has been reported that GZMA is involved in the COPD [59]. Notably, S100B and GZMA were associated with ALS-related signaling pathways. It has been demonstrated that muscle dysfunction is an important complication for COPD patients. Generally, COPD patients showed greater susceptibility muscle fatigue of the lower limb compared to healthy people [60-62]. Moreover, muscle mass loss or atrophy usually lead to the muscle dysfunction in COPD patients [34, 63]. Thus, GEM, S100B, and GZMA might be involved in the muscle dysfunction for COPD patients by ALS-related signaling pathways and might participate in the inflammatory response in COPD by regulating macrophage polarization, and these genes might act as the potential therapeutic targets for muscle atrophy of COPD patients.

Interestingly, GEM was significantly negatively correlated with $B L K$ and MICAL3, which are the two marker molecules for B cells, and $S 100 B$ was significantly positively correlated with the GNG7 marker for B cells (Figure 3(c)). Moreover, we found that GEM was highly associated with the genes in the PI3K/Akt/GSK3 $\beta$ signaling pathway, regulation of actin cytoskeleton, and calcium signaling pathway and might be partially related to the NF- $\kappa \mathrm{B}$ signaling pathway (Figure 4(a)). Especially, GEM was related to the $A k t$ in the PPI network (Figure 5(b)). These findings suggested that $G E M$ and $S 100 B$ might participate in the progression of COPD via regulating the activity of $\mathrm{B}$ cells and GEM might participate in the regulation of the PI3K/Akt/GSK3 $\beta$ signaling pathway. Apart from the macrophages, T lymphocytes also played an important role in COPD [64]. For example, Gosman et al. revealed that the number of B cells in bronchial biopsies of COPD was increased [65]. It has been revealed that glycyrrhizic acid could alleviate inflammatory lung disease, including chronic obstructive pulmonary disease by promoting the downstream PI3K/Akt/GSK3 $\beta$ signaling pathway [66]. Furthermore, compelling evidence has suggested that NF- $\kappa \mathrm{B}$ signaling plays a key role in the airway inflammation, including COPD [67]. Kim et al. also found that PI3K/Akt and NF- $\kappa \mathrm{B}$ signaling pathways were involved in the emphysematous change in COPD [68]. Taken together, these findings further suggested that $S 100 B$ and GEM might serve as the therapeutic target and might become the significant targets for drug identification and drug designing of COPD.
Finally, we found GEM could more precisely distinguish COPD from normal samples than the LASSO model obtained by combined GEM, S100B, and GZMA (Figure 6(c)). Hence, GEM could act as an independent diagnostic biomarker for COPD.

\section{Conclusions}

In summary, our study for the first time analyzes the role of macrophage polarization-related genes in COPD by WGCNA. In the present study, we found that the GEM, $S 100 B$, and GZMA might be the novel therapeutic targets for COPD and GEM could act as an independent diagnostic biomarker for COPD. Our findings may contribute to further understanding the molecular mechanism and improving the clinical diagnosis and treatment for COPD. However, there are still many limitations in this study, for instance, sample limitation and experiment limitation. Therefore, further experimental studies are essential for further verifying the functions and mechanisms of GEM, S100B, and GZMA in COPD.

\section{Data Availability}

The GSE124180 dataset analyzed in this study was available in the GEO database.

\section{Conflicts of Interest}

The authors declare that they have no conflicts of interest.

\section{Authors' Contributions}

Chaofeng Ren conceived and designed the research. Yalin Zhao, Meihua Li, Yanxia Yang, Tao Wu, Qingyuan Huang, and Qinghua Wu downloaded and analyzed the data. Yalin Zhao wrote the draft manuscript, and all authors approved the manuscript for publication.

\section{Acknowledgments}

The work was supported by the Kunming Science and Technology Bureau (2020-1-N-026).

\section{Supplementary Materials}

Supplementary Table 1: 35 macrophage polarization marker genes from the MsigDB database. Supplementary Table 2: 4922 genes in black module. Supplementary Table 3: 82 upregulated genes in COPD samples compared to normal samples. Supplementary Table 4: 121 downregulated genes in COPD samples compared to normal samples. Supplementary Table 5: overlapping genes between DEGs and macrophage polarization-related genes. (Supplementary Materials)

\section{References}

[1] P. J. Barnes, P. G. Burney, E. K. Silverman et al., "Chronic obstructive pulmonary disease," Nature Reviews Disease Primers, vol. 1, no. 1, p. 76, 2015. 
[2] C. F. Vogelmeier, G. J. Criner, F. J. Martinez et al., "Global strategy for the diagnosis, management, and prevention of chronic obstructive lung disease 2017 report: GOLD executive summary," European Respiratory Journal, vol. 49, no. 3, article 1700214, 2017.

[3] Organization WH, “The top 10 causes of death 2018," 2018, https://www.who.int/news-room/fact-sheets/detail/the-top10-causes-of-death.

[4] World Health Organization, "Global Health Estimates 2016," in Deaths by cause, age, sex, by country and by region, 20002016, World Health Organization, Geneva, Switzerland, 2018, 2019, https://www.who.int/healthinfo/global_burden_ disease/estimates/en/index1.html.

[5] A. S. Buist, W. M. Vollmer, and M. A. McBurnie, "Worldwide burden of COPD in high- and low-income countries. Part I. The burden of obstructive lung disease (BOLD) initiative," International Journal of Tuberculosis and Lung Disease, vol. 12, no. 7, pp. 703-708, 2008.

[6] A. Anzueto, "Impact of exacerbations on COPD," European Respiratory Review, vol. 19, no. 116, pp. 113-118, 2010.

[7] B. R. Celli, W. MacNee, A. Agusti et al., "Standards for the diagnosis and treatment of patients with COPD: a summary of the ATS/ERS position paper," European Respiratory Journal, vol. 23, no. 6, pp. 932-946, 2004.

[8] N. M. Alotaibi, V. Chen, Z. Hollander et al., "Phenotyping COPD exacerbations using imaging and blood-based biomarkers," International Journal of Chronic Obstructive Pulmonary Disease, vol. 13, pp. 217-229, 2018.

[9] F. Beckers, N. Lange, A. Koryllos, F. Picchioni, W. Windisch, and E. Stoelben, "Unilateral lobe resection by video-assisted thoracoscopy leads to the most optimal functional improvement in severe emphysema," The Thoracic and Cardiovascular Surgeon, vol. 64, no. 4, pp. 336-342, 2016.

[10] T. Sant'anna, N. A. Hernandes, and F. Pitta, "Pulmonary rehabilitation and COPD: is nonlinear exercise better?," Expert Review of Respiratory Medicine, vol. 7, no. 4, pp. 323-325, 2013.

[11] D. Singh, S. M. Fox, R. Tal-Singer, S. Bates, J. H. Riley, and B. Celli, "Altered gene expression in blood and sputum in COPD frequent exacerbators in the ECLIPSE cohort," PLoS One, vol. 9, no. 9, article e107381, 2014.

[12] W. Chen, Y. Q. Hong, and Z. L. Meng, "Bioinformatics analysis of molecular mechanisms of chronic obstructive pulmonary disease," European Review for Medical and Pharmacological Sciences, vol. 18, no. 23, pp. 357-363, 2014.

[13] J. Zhang, C. Zhu, H. Gao et al., "Identification of biomarkers associated with clinical severity of chronic obstructive pulmonary disease," PeerJ, vol. 8, article e10513, 2020.

[14] J. Zhao, W. Cheng, X. He et al., "Chronic obstructive pulmonary disease molecular subtyping and pathway deviationbased candidate gene identification," Cell Journal, vol. 20, no. 3, pp. 326-332, 2018.

[15] P. J. Barnes, "Inflammatory mechanisms in patients with chronic obstructive pulmonary disease," Journal of Allergy and Clinical Immunology, vol. 138, no. 1, pp. 16-27, 2016.

[16] U. Costabel and J. Guzman, "Effect of smoking on bronchoalveolar lavage constituents," European Respiratory Journal, vol. 5, no. 7, pp. 776-779, 1992.

[17] S. L. Traves, S. V. Culpitt, R. E. Russell, P. J. Barnes, and L. E. Donnelly, "Increased levels of the chemokines GROalpha and MCP-1 in sputum samples from patients with COPD," Thorax, vol. 57, no. 7, pp. 590-595, 2002.
[18] M. S. Eapen, P. M. Hansbro, K. McAlinden et al., "Abnormal M1/M2 macrophage phenotype profiles in the small airway wall and lumen in smokers and chronic obstructive pulmonary disease (COPD)," Scientific Reports, vol. 7, no. 1, article 13392, 2017.

[19] N. Li, Y. Liu, and J. Cai, "LncRNA MIR155HG regulates M1/M2 macrophage polarization in chronic obstructive pulmonary disease," Biomedicine \& Pharmacotherapy, vol. 117, article 109015, 2019.

[20] R. Shaykhiev, A. Krause, J. Salit et al., "Smoking-dependent reprogramming of alveolar macrophage polarization: implication for pathogenesis of chronic obstructive pulmonary disease," The Journal of Immunology, vol. 183, no. 4, pp. 28672883, 2009.

[21] J. A. Miller, R. L. Woltjer, J. M. Goodenbour, S. Horvath, and D. H. Geschwind, "Genes and pathways underlying regional and cell type changes in Alzheimer's disease," Genome Medicine, vol. 5, no. 5, p. 48, 2013.

[22] Z. Li, Y. Cui, J. Feng, and Y. Guo, "Identifying the pattern of immune related cells and genes in the peripheral blood of ischemic stroke," Journal of Translational Medicine, vol. 18, no. 1, pp. 1-17, 2020.

[23] E. Radulescu, A. E. Jaffe, R. E. Straub et al., "Identifcation and prioritization of gene sets associated with schizophrenia risk by co-expression network analysis in human brain," Molecular Psychiatry, vol. 25, pp. 791-804, 2018.

[24] T. Barrett, D. B. Troup, S. E. Wilhite et al., "NCBI GEO: mining tens of millions of expression profiles-database and tools update," Nucleic Acids Research, vol. 35, pp. D760-D765, 2007.

[25] P. Langfelder and S. Horvath, "WGCNA: an R package for weighted correlation network analysis," BMC Bioinformatics, vol. 9, no. 1, p. 559, 2008.

[26] A. L. Barabási and E. Bonabeau, "Scale-free networks," Scientific American, vol. 288, no. 5, pp. 60-69, 2003.

[27] B. Zhang and S. Horvath, "A general framework for weighted gene co-expression network analysis," Statistical Applications in Genetics and Molecular Biology, vol. 4, no. 1, p. 17, 2005.

[28] P. Langfelder, B. Zhang, and S. Horvath, "Defining clusters from a hierarchical cluster tree: the dynamic tree cut package for R,” Bioinformatics, vol. 24, no. 5, pp. 719-720, 2008.

[29] S. Anders and W. Huber, "Differential expression analysis for sequence count data," Genome Biology, vol. 11, no. 10, p. R106, 2010.

[30] Z. Bozdech, J. Zhu, M. P. Joachimiak, F. E. Cohen, B. Pulliam, and J. L. DeRisi, "Expression profiling of the schizont and trophozoite stages of Plasmodium falciparum with a longoligonucleotide microarray," Genome Biology, vol. 4, no. 2, p. R9, 2003.

[31] I. S. Ramsay, S. Ma, M. Fisher et al., "Model selection and prediction of outcomes in recent onset schizophrenia patients who undergo cognitive training," Schizophrenia Research: Cognition, vol. 11, pp. 1-5, 2018.

[32] X. Robin, N. Turck, A. Hainard et al., "pROC: an open-source package for R and S+ to analyze and compare ROC curves," BMC Bioinformatics, vol. 12, no. 1, p. 77, 2011.

[33] S. C. Funes, M. Rios, J. Escobar-Vera, and A. M. Kalergis, "Implications of macrophage polarization in autoimmunity," Immunology, vol. 154, no. 2, pp. 186-195, 2018.

[34] R. C. Wüst and H. Degens, "Factors contributing to muscle wasting and dysfunction in COPD patients," International Journal of Chronic Obstructive Pulmonary Disease, vol. 2, no. 3, pp. 289-300, 2007. 
[35] G. Bindea, B. Mlecnik, M. Tosolini et al., "Spatiotemporal dynamics of intratumoral immune cells reveal the immune landscape in human cancer," Immunity, vol. 39, no. 4, pp. 782-795, 2013.

[36] A. A. Gladkikh, D. M. Potashnikova, V. J. Tatarskiy et al., "Comparison of the mRNA expression profile of B-cell receptor components in normal CD5-high B-lymphocytes and chronic lymphocytic leukemia: a key role of ZAP70," Cancer Medicine, vol. 6, no. 12, pp. 2984-2997, 2017.

[37] C. F. Vogelmeier, G. J. Criner, F. J. Martinez et al., "Global strategy for the diagnosis, management, and prevention of chronic obstructive lung disease 2017 report: GOLD executive summary," Archvosde Bronconeumologia, vol. 53, pp. 128-149, 2017.

[38] G. A. Roth, D. Abate, K. H. Abate et al., "Global, regional, and national age-sex-specific mortality for 282 causes of death in 195 countries and territories, 1980-2017: a systematic analysis for the Global Burden of Disease Study 2017," The Lancet, vol. 392, no. 10159, pp. 1736-1788, 2018.

[39] H. Wang, M. Naghavi, C. Allen et al., "GBD 2015 Mortality and Causes of Death Collaborators (2016) Global, regional, and national life expectancy, all-cause mortality, and causespecific mortality for 249 causes of death, 1980-2015: a systematic analysis for the Global Burden of Disease Study 2015," The Lancet, vol. 388, no. 10053, pp. 1459-1544, 2016.

[40] Q. Song, D. C. Christiani, XiaorongWang, and J. Ren, “The global contribution of outdoor air pollution to the incidence, prevalence, mortality and hospital admission for chronic obstructive pulmonary disease: a systematic review and metaanalysis," International Journal of Environmental Research and Public Health, vol. 11, no. 11, pp. 11822-11832, 2014.

[41] F. Cortopassi, P. Gurung, and V. Pinto-Plata, "Chronic obstructive pulmonary disease in elderly patients," Clinics in Geriatric Medicine, vol. 33, no. 4, pp. 539-552, 2017.

[42] J. D. Morrow, R. P. Chase, M. M. Parker et al., "RNA-sequencing across three matched tissues reveals shared and tissuespecific gene expression and pathway signatures of COPD," Respiratory Research, vol. 20, no. 1, p. 65, 2019.

[43] E. E. Baschal, E. D. Larson, T. C. Bootpetch Roberts et al., "Identification of novel genes and biological pathways that overlap in infectious and nonallergic diseases of the upper and lower airways using network analyses," Frontiers in Genetics, vol. 10, p. 1352, 2020.

[44] A. A. Lambert, N. Putcha, M. B. Drummond et al., "Obesity is associated with increased morbidity in moderate to severe COPD," Chest, vol. 151, no. 1, pp. 68-77, 2017.

[45] C. Hanson, E. P. Rutten, E. F. Wouters, and S. Rennard, "Influence of diet and obesity on COPD development and outcomes," International Journal of Chronic Obstructive Pulmonary Disease, vol. 9, pp. 723-733, 2014.

[46] Y. Li, J. Shi, J. Yang et al., “A Wnt/ $\beta$-catenin negative feedback loop represses TLR-triggered inflammatory responses in alveolar epithelial cells," Molecular, Immunology, vol. 59, no. 2, pp. 128-135, 2014.

[47] K. Schaale, J. Neumann, D. Schneider, S. Ehlers, and N. Reiling, "Wnt signaling in macrophages: augmenting and inhibiting mycobacteria-induced inflammatory responses," European Journal of Cell Biology, vol. 90, no. 6-7, pp. 553559, 2011.

[48] H. R. Bourne, D. A. Sanders, and F. McCormick, "The GTPase superfamily: conserved structure and molecular mechanism," Nature, vol. 349, no. 6305, pp. 117-127, 1991.
[49] J. Maguire, T. Santoro, P. Jensen, U. Siebenlist, J. Yewdell, and K. Kelly, "Gem: an induced, immediate early protein belonging to the Ras family," Science, vol. 265, no. 5169, pp. 241-244, 1994.

[50] R. Bianchi, E. Kastrisianaki, I. Giambanco, and R. Donato, "S100B protein stimulates microglia migration via RAGEdependent up-regulation of chemokine expression and release," Journal of Biological Chemistry, vol. 286, no. 9, pp. 7214-7226, 2011.

[51] F. Riuzzi, S. Beccafico, R. Sagheddu et al., "Levels of S100B protein drive the reparative process in acute muscle injury and muscular dystrophy," Scientific Reports, vol. 7, no. 1, article 12537, 2017.

[52] A. Fujiya, H. Nagasaki, Y. Seino et al., "The role of S100B in the interaction between adipocytes and macrophages," Obesity, vol. 22, no. 2, pp. 371-379, 2014.

[53] L. Zhu, Z. Weng, P. Shen et al., "S100B regulates inflammatory response during osteoarthritis via fibroblast growth factor receptor 1 signaling," Molecular Medicine Reports, vol. 18, no. 6, pp. 4855-4864, 2018.

[54] N. Ohtaki, W. Kamitani, Y. Watanabe et al., "Downregulation of an astrocyte-derived inflammatory protein, S100B, reduces vascular inflammatory responses in brains persistently infected with Borna disease virus," Journal of Virology, vol. 81, no. 11, pp. 5940-5948, 2007.

[55] L. Santiago, C. Menaa, M. Arias et al., "Granzyme A contributes to inflammatory arthritis through stimulation of osteoclastogenesis," Arthritis \& Rheumatology, vol. 69, pp. 320334, 2017.

[56] J. A. Wilson, N. A. Prow, W. A. Schroder et al., "RNA-Seq analysis of chikungunya virus infection and identification of granzyme a as a major promoter of arthritic inflammation," PLoS Pathogens, vol. 13, no. 2, article e1006155, 2017.

[57] G. W. Tew, J. A. Hackney, D. Gibbons et al., "Association between response to etrolizumab and expression of integrin alphaE and granzyme A in colon biopsies of patients with ulcerative colitis," Gastroenterology, vol. 150, no. 2, pp. 477487.e9, 2016.

[58] M. Garzón-Tituaña, J. L. Sierra-Monzón, L. Comas et al., "Granzyme A inhibition reduces inflammation and increases survival during abdominal sepsis," Theranostics, vol. 11, no. 8, pp. 3781-3795, 2021.

[59] J. H. Vernooy, G. M. Möller, R. J. van Suylen et al., "Increased granzyme A expression in type II pneumocytes of patients with severe chronic obstructive pulmonary disease," American Journal of Respiratory and Critical Care Medicine, vol. 175, no. 5, pp. 464-472, 2007.

[60] D. Bachasson, B. Wuyam, J. L. Pepin, R. Tamisier, P. Levy, and S. Verges, "Quadriceps and respiratory muscle fatigue following high-intensity cycling in COPD patients," PLoS One, vol. 8, no. 12, article e83432, 2013.

[61] C. Burtin, D. Saey, M. Saglam et al., "Effectiveness of exercise training in patients with COPD: the role of muscle fatigue," European Respiratory Journal, vol. 40, no. 2, pp. 338-344, 2012.

[62] C. Coronell, M. Orozco-Levi, R. Mendez, A. Ramirez-Sarmiento, J. B. Galdiz, and J. Gea, "Relevance of assessing quadriceps endurance in patients with COPD," European Respiratory Journal, vol. 24, no. 1, pp. 129-136, 2004.

[63] E. Barreiro and A. Jaitovich, "Muscle atrophy in chronic obstructive pulmonary disease: molecular basis and potential 
therapeutic targets," Journal of Thoracic Disease, vol. 10, Supplement 12, pp. S1415-S1424, 2018.

[64] C. Donovan, M. R. Starkey, R. Y. Kim et al., "Roles for T/B lymphocytes and ILC2s in experimental chronic obstructive pulmonary disease," Journal of Leukocyte Biology, vol. 105, no. 1, pp. 143-150, 2019.

[65] M. M. Gosman, B. W. Willemse, D. F. Jansen et al., "Increased number of B-cells in bronchial biopsies in COPD," European Respiratory Journal, vol. 27, no. 1, pp. 60-64, 2006.

[66] T. C. Kao, M. H. Shyu, and G. C. Yen, "Glycyrrhizic acid and $18 \beta$-glycyrrhetinic acid inhibit inflammation via PI3K/Akt/GSK3 $\beta$ signaling and glucocorticoid receptor activation," Journal of Agricultural and Food Chemistry, vol. 58, no. 15, pp. 8623-8629, 2010.

[67] M. Schuliga, "NF-kappaB signaling in chronic inflammatory airway disease," Biomolecules, vol. 5, no. 3, pp. 1266-1283, 2015.

[68] S. E. Kim, T. T. T. Thuy, J. H. Lee et al., "Simvastatin inhibits induction of matrix metalloproteinase- 9 in rat alveolar macrophages exposed to cigarette smoke extract," Experimental \& Molecular Medicine, vol. 41, no. 4, pp. 277-287, 2009. 